\title{
ÍNDICE SPAD E GRAU BRIX DA CULTURA DO RABANETE SOB LÂMINAS DE ÁGUA E DOSES DE ADUBAÇÃO NITROGENADA
}

\author{
Floriano Alcantara Damasceno ${ }^{1 *}$, Danilo Santos Silva ${ }^{2}$, Kívia Caroline da Costa ${ }^{2}$, Allyne Hariadrinny Pereira \\ Barbosa $^{2}$, Julianna Catonio da Silva ${ }^{3}$, Márcio Aurélio Lins dos Santos ${ }^{4}$ \\ 'Universidade Federal de Alagoas, Campus de Arapiraca. Av. Manoel Severino Barbosa, Arapiraca, Alagoas, CEP 57309-005 *Fone: \\ (82)9 99612-0768. E-mail: floriano23@hotmail.com. \\ 2Universidade Federal de Alagoas, Campus de Arapiraca. \\ ${ }^{3}$ Universidade Federal de Alagoas - Campus de Engenharias e de Ciências Agrárias. \\ ${ }^{4}$ Universidade Federal de Alagoas, Campus de Arapiraca.
}

\begin{abstract}
RESUMO: O rabanete (Raphanus sativus L.) exige boa disponibilidade hídrica e fertilidade do solo. O manejo da irrigação é indispensável, assim como adubação. Objetivou-se avaliar a cultura do rabanete em função de diferentes lâminas de irrigação e doses de adubação nitrogenada. 0 experimento foi realizado na Universidade Federal de Alagoas, Campus de Arapiraca com delineamento em blocos casualizados em fatorial $5 \times 5$ com quatro repetições. Os tratamentos consistiram em cinco lâminas de água com 50\%,75\%,100\%, 125\% e 150\% da evapotranspiração da cultura, com cinco doses de adubação nitrogenada correspondendo a 50\%,75\%,100\%, 125\%, e 150\% da recomendação para a cultura. As variáveis analisadas foram o índice SPAD e o grau BRIX. Para o grau BRIX foi significativo apenas para o fator lâmina com um com comportamento decrescente e um ponto mínimo de 3,82\% na lâmina de 196,67 mm. Já a variável índice SPAD foi significativa para a interação entre os dois fatores, com 0 ponto máximo na lâmina de 187,53 mm e adubação de 45,43 Kgha-1. A lâmina de irrigação interfere no grau de BRIX do rabanete e o índice SPAD é influenciado pela irrigação e adubação nitrogenada.
\end{abstract}

PALAVRAS CHAVE: Raphanus sativus, manejo de irrigação, nitrogênio.

\section{SPAD INDEX AND BRIX DEGREE OF RABANET CULTURE UNDER WATER BLADES AND NITROGEN FERTILIZATION DOSES}

\begin{abstract}
The radish (Raphanus sativus L.) requires good water availability and soil fertility. Irrigation management is essential, as well as fertilization. The objective was to evaluate the radish culture according to different irrigation depths and nitrogen fertilization doses. The experiment was carried out at the Federal University of Alagoas, Arapiraca Campus with a randomized block design in a $5 \times 5$ factorial with four repetitions. The treatments consisted of five water slides with $50 \%, 75 \%, 100 \%, 125 \%$ and $150 \%$ of the evapotranspiration of the culture, with five doses of nitrogen fertilization corresponding to $50 \%, 75 \%, 100 \%, 125 \%$, and $150 \%$ of recommendation for culture. The variables analyzed were the SPAD index and the BRIX degree. For the BRIX degree, it was significant only for the blade factor with one with decreasing behavior and a minimum point of $3.82 \%$ on the $196.67 \mathrm{~mm}$ blade. The SPAD index variable was significant for the interaction between the two factors, with the maximum point on the blade of $187.53 \mathrm{~mm}$ and fertilization of $45.43 \mathrm{Kgha}-1$. The irrigation depth interferes with the BRIX degree of the radish and the SPAD index is influenced by irrigation and nitrogen fertilization.
\end{abstract}

KEY WORDS: Raphanus sativus, irrigation management, nitrogen

\section{INTRODUÇÃO}

O rabanete (Raphanus sativus L.) é uma olerícola pertencente à família Brassicaceae, consisti em uma planta de pequeno porte, possuindo uma raiz carnuda, no formato globular, ovoide ou alongado e coloração escarlate brilhante com polpa branca (Filgueira, 2008). 0 mesmo autor destaca que esta cultura vem sendo difundida entre os produtores, pois possui características desejáveis, como seu ciclo curto, boa rusticidade e a colheita podendo ser realizada entre 25 e 30 dias após 0 plantio. 
$\mathrm{Na}$ agricultura, o cultivo de hortaliças é considerado uma das áreas mais exigentes do ponto de vista nutricional e hídrica do solo, desta forma, a utilização de técnicas adequadas de adubação e irrigação são alternativas indispensáveis para 0 aumento na produtividade e diminuição dos riscos na atividade (Bernardino et al., 2017). Por ser uma cultura onde o produto a ser comercializado fica localizado de forma subsuperficial no solo, o rendimento do rabanete pode ser influenciado diretamente pelas condições físicohídricas do solo (Silva et al., 2012).

Dentre os recursos necessários para 0 metabolismo da planta, a água é considerada o mais abundante e em grande parte 0 mais limitante para a produção agrícola. Desta forma, a prática da irrigação das culturas reflete no fato de que a água é um recursochave capaz de limitar a produtividade agrícola (Taiz etal., 2017). Com isso é indispensável o uso racional da água, enfatizando a aplicação em quantidades adequadas. Além disso, é necessária a avaliação dos fatores que compõe os sistemas de produção, relacionados a eficiência e o manejo da irrigação (Dantas et al., 2014).

Além da irrigação, outro fator importante e indispensável para a produção hortícola é a adubação, visto que a falta ou o excesso de um nutriente mineral torna-se um fator limitante para a espécie vegetal cultivada. $O$ nitrogênio é o elemento mineral que as plantas exigem em maiores quantidades, servindo como constituinte de muitos componentes celulares vegetais, incluindo clorofila, aminoácidos e ácidos nucleicos.com isso, a deficiência de nitrogênio inibe rapidamente o crescimento vegetal (Taiz et al., 2017).

Objetivou-se avaliar a cultura do rabanete em função de diferentes lâminas de irrigação e doses de adubação nitrogenada.

\section{MATERIAL E MÉTODOS}

0 experimento foi conduzido na área experimental da Universidade Federal de Alagoas, Campus de Arapiraca, em ambiente protegido (casa de vegetação), situada latitude $9^{\circ} 45^{\prime} 09^{\prime \prime}$ Sul e longitude $36^{\circ} 39^{\prime} 40^{\prime \prime}$ Oeste, com altitude de $325 \mathrm{~m}$. 0 ensaio estatístico ocorreu no período de 02 de abril a 02 de maio de 2018, sendo início da estação chuvosa com temperaturas mais baixas.

0 delineamento experimental utilizado foi em blocos casualizados (DBC), em esquema fatorial
$5 \times 5$ com quatro repetições. As lâminas de irrigação utilizadas foram obtidas através da lisimetria de drenagem, realizando-se leituras diárias para obtenção da ETc (evapotranspiração da cultura) através da diferença entre a lâmina aplicada e drenada, onde consistiram em L1-50\% do ETc $(71,77 \mathrm{~mm})$, L2-75\% do ETc $(107,65 \mathrm{~mm})$, L3- $100 \%$ da ETc $(143,54 \mathrm{~mm})$, L4-125\% da ETc $(179,42 \mathrm{~mm})$ e L5-150\% da ETC $(215,3 \mathrm{~mm})$.

O segundo fator utilizado foi adubação química nitrogenada de fundação e cobertura. Onde a fonte de nitrogênio utilizada foi ureia, com $45 \%$ de N. Os tratamentos consistiram em 50,75, 100,125, e 150\% da recomendação para a cultura do Instituto Agronômico de Pernambuco (2008) que é correspondente respectivamente a 20, 30, 40, 50 e $60 \mathrm{Kgha}^{-1}$. Além desses tratamentos procedeu-se a adubação com os demais nutrientes conforme a recomendação para todas as parcelas.

Para o plantio do rabanete foi utilizado em recipientes de polietileno com capacidade de 5,5 L e diâmetro de 0,20 m. 0 substrato para o cultivo foi solo arenoso, onde previamente realizou-se análise química do mesmo. Na obtenção da evapotranspiração da cultura (ETc) utilizou-se lisímetros de drenagem, sendo cinco recipientes plásticos idênticos às parcelas experimentais, diferenciando-se somente por conter drenos e reservatórios.

A ETc foi determinada diariamente por meio da coleta dos valores drenados pelos lisímetros, onde eram descartados os dois valores mais discrepantes (maior e menor) e aferida a média aritmética dos demais valores que era subtraído pelo valor aplicado.

O plantio ocorreu diretamente no solo em capacidade de campo, sendo antecedido pelo preparo do mesmo com uma adubação de fundação. E a colheita ocorreu 30 dias após o plantio. As variáveis avaliadas foram os sólidos solúveis totais ( $\left.{ }^{\circ} \mathrm{BRIX}\right)$ por meio do refratômetro, e o teor relativo de clorofila índice SPAD por meio do Clorofilômetro digital SPAD 502. Os dados experimentais foram submetidos a análise de variância com teste de regressão no software estatístico $R$ i386 3.5.1.

\section{RESULTADOS E DISCUSSÃO}

De acordo com a tabela de análise de variância (Tabela 1) houve efeito significativo para o fator índice 
SPAD e grau BRIX. Para adubação nitrogenada foi a interação entre os fatores foi possível observar a significativo somente para 0 índice SPAD. Já para interação também somente para o índice SPAD.

Tabela 1. Resumo de análise de variância para grau BRIX e índice SPAD.

\begin{tabular}{lccc}
\hline & GL & \multicolumn{2}{c}{ VALORES DE QUADRADOS MÉDIOS } \\
\cline { 3 - 4 } & & SPAD & BRIX \\
\cline { 3 - 4 } Lâminas & 4 & $422.119^{*}$ & $3.649^{*}$ \\
Adubação & 4 & $59.842^{*}$ & $0.233^{\text {Ns }}$ \\
Lâminas x Adubação & 16 & $26.102^{*}$ & $0.403^{\text {Ns }}$ \\
Blocos & 3 & 4.599 & 0.463 \\
Resíduo & 72 & 4.504 & 0.274 \\
\hline CV $(\%)$ & & 4.97 & 12.80 \\
\hline
\end{tabular}

${ }^{*}$ significativo a $5 \%$ de probabilidade, Ns não significativo.

A variável sólidos solúveis totais ( ${ }^{\circ} \mathrm{BRIX}$ $\%$ ), apresentou um comportamento de regressão quadrática de forma decrescente sendo inversa a lâmina de água, ou seja, quanto maior a lâmina menor o BRIX e quanto menor a lâmina de água, maior 0 BRIX. Seu ponto mínimo do grau de ${ }^{\circ}$ BRIX ocorreu na lâmina de 196,67 mm, obtendo-se a média de
$3,82 \%^{\circ} \mathrm{BRIX}$ (Figura 01), isso pode estar relacionado ao potencial hídrico das células e a concentração de solutos nas mesmas, pois em plantas que recebem mais água a concentração de solutos nas células tendem a ser menor do que em plantas com déficit hídrico que possui um potencial osmótico maior (Taiz et al., 2017).

Figura 1. Valores de sólidos solúveis totais $\left({ }^{\circ} \mathrm{BRIX} \%\right)$ do rabanete em função da lâmina de água.

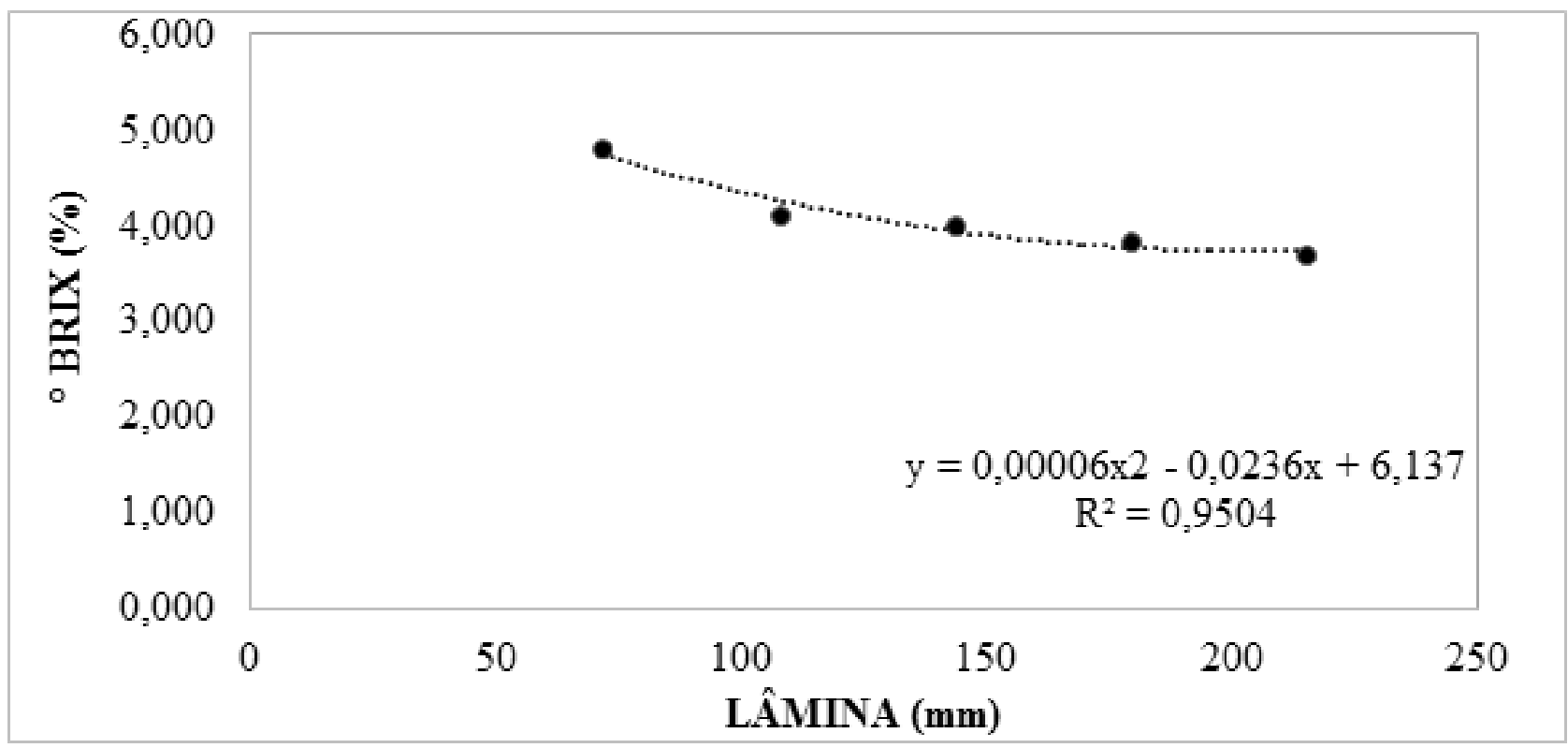

Barbosa Júnior et al., (2017) trabalhando com a mesma cultura obtiveram resultados semelhantes, uma vez que o grau BRIX foi menor em lâminas de água superiores e consequentemente valores mais elevados em lâminas de água inferiores. Isso foi atribuído pelo autor ao fato que os bulbos oriundos do tratamento com a menor lâmina de água, por sua vez, apresentaram menor desenvolvimento, entretanto maior valor de BRIX, o que implica em dizer que bulbos menores apresentam uma maior concentração de sais.
A variável índice SPAD que mensura o teor relativo de clorofila apresentou interação significativa ente os fatores lâmina e adubação nitrogenada. Para o fator lâmina teve comportamento decrescente, com isso diminuindo conforme a lâmina de água aplicada aumentou. E em relação ao fator adubação, o índice SPAD foi crescente, ou seja, conforme aumentou a dose de nitrogênio aplicada o teor relativo de clorofila também teve acréscimo conforme pode ser observado na Figura 2, o ponto máximo foi de 39,75 
ocorreu na lâmina de 187,53 mm com a adubação de $\quad 45,43$ Kg.ha-1.

Figura 2. Gráfico 3D para a variável SPAD com a interação entre lâminas de irrigação e doses de adubação nitrogenada. (Plotado em: <http://www.calculadoraonline.com.br/grafica-avancada>).

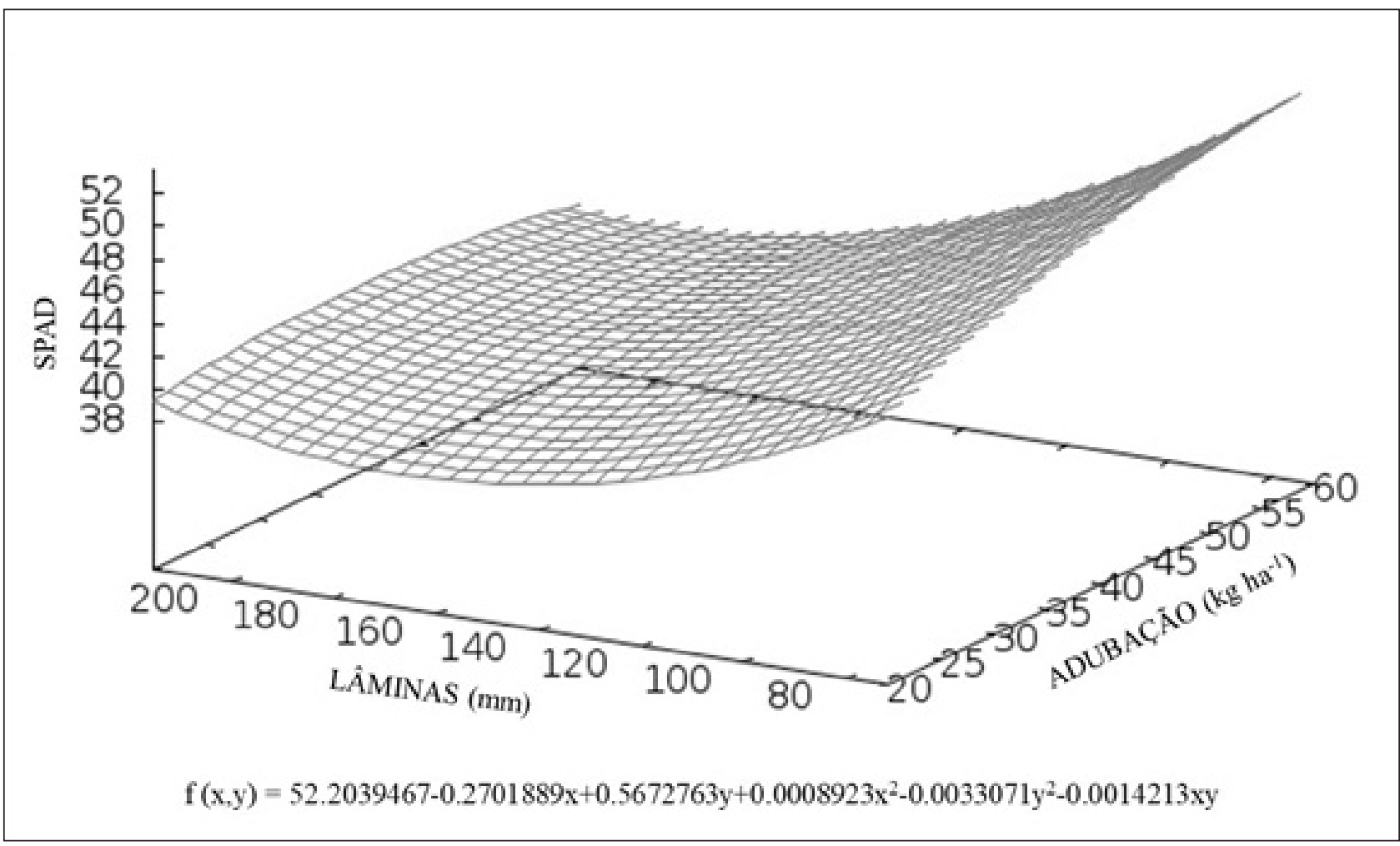

Trabalhando com a cultura do rabanete em condições análogas, Silva et al., (2017) encontrou resultados semelhantes, pois o índice SPAD obtido foi inversamente proporcional a lâmina de água aplicada, ou seja, apresentou comportamento decrescente com 0 aumento da lâmina de irrigação. Apesar de que 0 índice SPAD aumentou conforme a dose de adubação nitrogenada foi elevada, Quadros et al., (2010) constatou que para a cultura do rabanete não existe correlação significativa entre o teor relativo de clorofila e o nitrogênio na planta, pois segundo ele, boa parte do nitrogênio absorvido é provavelmente utilizado para a produção de outras estruturas na planta e não no acúmulo na folha para síntese da clorofila.

Desta forma, a lâmina de irrigação interfere diretamente no grau de BRIX do rabanete. 0 índice SPAD sofre influência de diferentes lâminas de irrigação aplicadas na cultura como também de diferentes doses de adubação nitrogenada.

\section{REFERÊNCIAS BIBLIOGRÁFICAS}

Barbosa Júnior, M. R.; Silva T. R. G.; Silva; J. C.; Silva, C. B.; Santos,
D. P.; Santos, M. A. L. Avaliação da cultura do rabanete em função de diferentes lâminas de água. IV INOVAGRI International Meeting, 2017.

Bernardino M. M., Alves, D. M., Dias J. H. R., Bastos, A. V. S., Santos,

L. N. S., Costa, C. T. S. Cultivo do rabanete cv. Vermelho gigante, submetido a reposições hídricas e fertirrigação com fontes de nitrogênio. IV INOVAGRI International Meeting, 2017.

Cavalcanti, F. J. A. Recomendações de adubação para o Estado de Pernambuco: segunda aproximação. Instituto Agronômico de Pernambuco, IPA. 212p. 2008.

Dantas, I. L. A.; Faccioli, L. G. G.; Nunes, L. P.; Viegas, T. A. P, Viabilidade

do uso de água residuária tratada na irrigação da cultura do rabanete (Raphanus sativus L.). Revista Ambiente e Água, 2014, 9, 1, 109-117. 
Filgueira, F. A. R. Novo manual de olericultura: medidor portátil nas folhas. Irriga, 2010, 15, 4, 353-360. Agrotecnologia moderna na produção e comercialização de hortaliças. Viçosa-MG: UFV, 2008. 421 p.

Silva, R. T.; Souza, A. A. T.; Oliveira, F. A.; Targino, I. S. O.; Silva, M. L.N. Tolerância do rabanete ao Quadros, B. R.; Silva, E. S.; Borges, L. S.; Moreira, C. $\quad$ encharcamento do solo. Revista Verde, 2012, 7, 1, 25A.; Moro, A. L.; 33.

Villas Bôas, R, L. Doses de nitrogênio na produção de Taiz, L. Fisiologia e desenvolvimento vegetal; 6. ed. rabanete fertirrigado e determinação de clorofila por Porto Alegre. 2017. 